\title{
Microstimulation of the Dorsolateral Prefrontal Cortex Biases Saccade Target Selection
}

\author{
Ioan Opris, Andrei Barborica, and Vincent P. Ferrera
}

\begin{abstract}
A long-standing issue concerning the executive function of the primate dorsolateral prefrontal cortex is how the activity of prefrontal neurons is linked to behavioral response selection. To establish a functional relationship between prefrontal memory fields and saccade target selection, we trained three macaque monkeys to make saccades to the remembered location of a visual cue in a delayed spatial match-to-sample saccade task. We electrically stimulated sites in the prefrontal cortex with subthreshold currents during the delay epoch
\end{abstract}

\section{INTRODUCTION}

Voluntary movement control depends on the ability to select spatial targets for future actions or to withhold a selected option (Miller \& Cohen, 2001; Rowe, Toni, Josephs, Frankowiak, \& Passingham, 2000; Hanes \& Schall, 1996; Posner, Rafal, Choate, \& Vaughan, 1985). Previous electrophysiological and imaging studies suggest a link between the executive mechanisms of behavioral selection and information encoded in the spatially selective delay activity of dorsolateral prefrontal cortical (dlPFC) neurons (Baddeley, 2003; Rowe et al., 2000; Courtney, Ungerleider, Keil, \& Haxby, 1997; Sweeney et al., 1996; Goldman-Rakic, 1995, 1996; Funahashi, Bruce, \& Goldman-Rakic, 1989; Fuster \& Alexander, 1971). Many neurons in the primate prefrontal cortex discharge while a stimulus is maintained on-line in working memory (Funahashi et al., 1989), suggesting that delay activity may be related to visual attention (Eveling, Tinsley, Gaffan, \& Duncan, 2002; Lebedev \& Wise, 2002), saccade preparation/planning (Opris, Barborica, \& Ferrera, submitted; Hanes \& Schall, 1996), and perceptual decision making (Barraclough, Conroy, \& Lee, 2004; Constantinidis, Franowicz, \& Goldman-Rakic, 2001; Kim \& Shadlen, 1999). Consistent with the hypothesized role in visual selection, the frontal eye field (FEF) appears to "gate" both the visual signals involved in attention, as well as the movement signals used for saccade preparation (Moore \& Armstrong, 2003; Burman \& Bruce, 1997; Schall, Hanes, Thompson,

Columbia University while monkeys performed this task. Our results show that the artificially injected signal interacts with the neural activity responsible for target selection, biasing saccade choices either towards the receptive/movement field (RF/MF) or away from the $\mathrm{RF} / \mathrm{MF}$, depending on the stimulation site. These findings might reflect a functional link between prefrontal signals responsible for the selection bias by modulating the balance between excitation and inhibition in the competitive interactions underlying behavioral selection.
\& King, 1995). Previous reports have shown that microstimulation biases perceptual judgments (Gold \& Shadlen, 2000; Seideman, Zohary, \& Newsome, 1998; Salzman \& Newsome, 1994; for review, see Cohen \& Newsome, 2004), affects decision speed (Ditterich, Mazurek, \& Shadlen, 2003), or disrupts target selection (Tehovnik \& Slocum, 2003). Yet, it has remained relatively unexplored whether altering delay period activity in the dlPFC can affect target selection behavior. The present study addresses the effects of prefrontal cortical microstimulation on saccade target selection by injecting a subthreshold microcurrent during the delay period of a spatial delayed response task. This approach differs from other studies in that we have replaced the perceptual discrimination/detection component of the task with a highly salient spatial cue that unambiguously signals the location of the target. This modification reduces uncertainty about the correct response and allows one to directly probe the role of the prefrontal cortex in specifying the behavioral response. A preliminary description of the results was presented as an abstract (Opris, Barborica, \& Ferrera, 2003).

\section{RESULTS}

To explore the functional relationship between prefrontal activity and saccade target selection, we trained monkeys to make saccades to the remembered location of the visual cue in two related cognitive tasks. For each experiment, we first recorded the activity of prefrontal neurons during a memory-guided saccade (MGS) task (Funahashi et al., 1989), and then electrically stimulated 
the same sites with subthreshold currents during the delay interval of a spatial match-to-sample (MTS) saccade task (Figure 1A and B). There were a total of 53 stimulation experiments, of which 26 were performed at low-threshold $(\leq 50 \mu \mathrm{A})$ FEF sites and 27 at nearby sites located on the anterior bank of the arcuate sulcus but having stimulation thresholds $>50 \mu \mathrm{A}$. The design of the MTS task comprised all combinations of three binary variables: stimulation (present, absent), target-distractor separation $\left(45^{\circ}, 180^{\circ}\right)$, and spatial cue (present, absent) for a total of eight conditions (see Figure 1). All conditions were randomly interleaved and each was repeated at least 10 times for a given stimulation site. A fixation task was used to determine the electrical saccade threshold and vector for sites where saccades could be evoked by microstimulation.

An example of the effect of subthreshold stimulation during the MTS task is illustrated in Figure 2A and B by showing raw eye position traces for the first saccade initiated after the end of the delay interval (total = 295 saccades). The neuron recorded at this site showed weak direction tuning during the memory-saccade task, however saccades could be evoked reliably by stimulating during fixation with a current of $30 \mu \mathrm{A}$. The mean electrically evoked saccade vector (amplitude $=$ $11^{\circ}$, direction $=260^{\circ}$ ) is indicated by the light gray arrow. The endpoint of this arrow is an estimate of the movement field (MF) of the stimulation site. During the MTS task, the monkey chose the correct target on about $80 \%$ of the trials in the absence of stimulation. When stimulation was applied during the delay interval (current $=15 \mu \mathrm{A}$ ), choice saccades initiated after the offset of stimulation were consistently biased toward the MF. This resulted in an increased percent correct when the correct target was in the MF (Figure 2A), and a decreased percentage when the target was at the location opposite the MF (Figure 2B). The statistical significance of the stimulation effect was determined using Cochran's Q-test for dichotomous outcomes. There were highly significant differences between stimulated and nonstimulated trials for both correct versus incorrect choices $\left(p<10^{-8}\right)$ and for choices toward or away from the MF $\left(p<10^{-12}\right)$. The effect of stimulation at this site for the target-distractor separation of $45^{\circ}$ was also significant for both percent correct $(p<.0001)$ and percent of choices toward the MF $(p<.0001)$. The stimulation effect in the absence of the spatial cue was marginally significant for both target-distractor separations $\left(45^{\circ}\right.$ and $180^{\circ}$ ) and outcome classes (percent correct and percent toward MF; $p<.03$ ).
Figure 1. Schematic

description of the microstimulation paradigm. (A) MTS saccade task with symmetrically opposite targets (B) Temporal sequence of the events in spatial MTS saccade task. At the beginning of each trial, the monkey fixates a small white square. A peripheral cue is flashed for $300 \mathrm{msec}$. Spatial cues were located either within the RF or at a location outside the RF (non-RF) with equal probability. The animal is required to maintain fixation during the entire delay epoch. At the end of the delay interval, the fixation spot disappears and two targets are presented for 500 msec. The monkey is then allowed to make a saccade to one of the targets. Throughout the delay (1000 msec), subthreshold currents $(<50 \mu \mathrm{A})$ of $350 \mathrm{~Hz}$ were applied in $50 \%$ of the trials (gray color).

(C) Configuration of cue and target distribution in the spatial MTS saccade task. There were three conditions for the cue: (i) cue in the RF, (ii) cue outside the RF, and (iii) no-cue, and two conditions for target separations: $180^{\circ}$ and $45^{\circ}$.
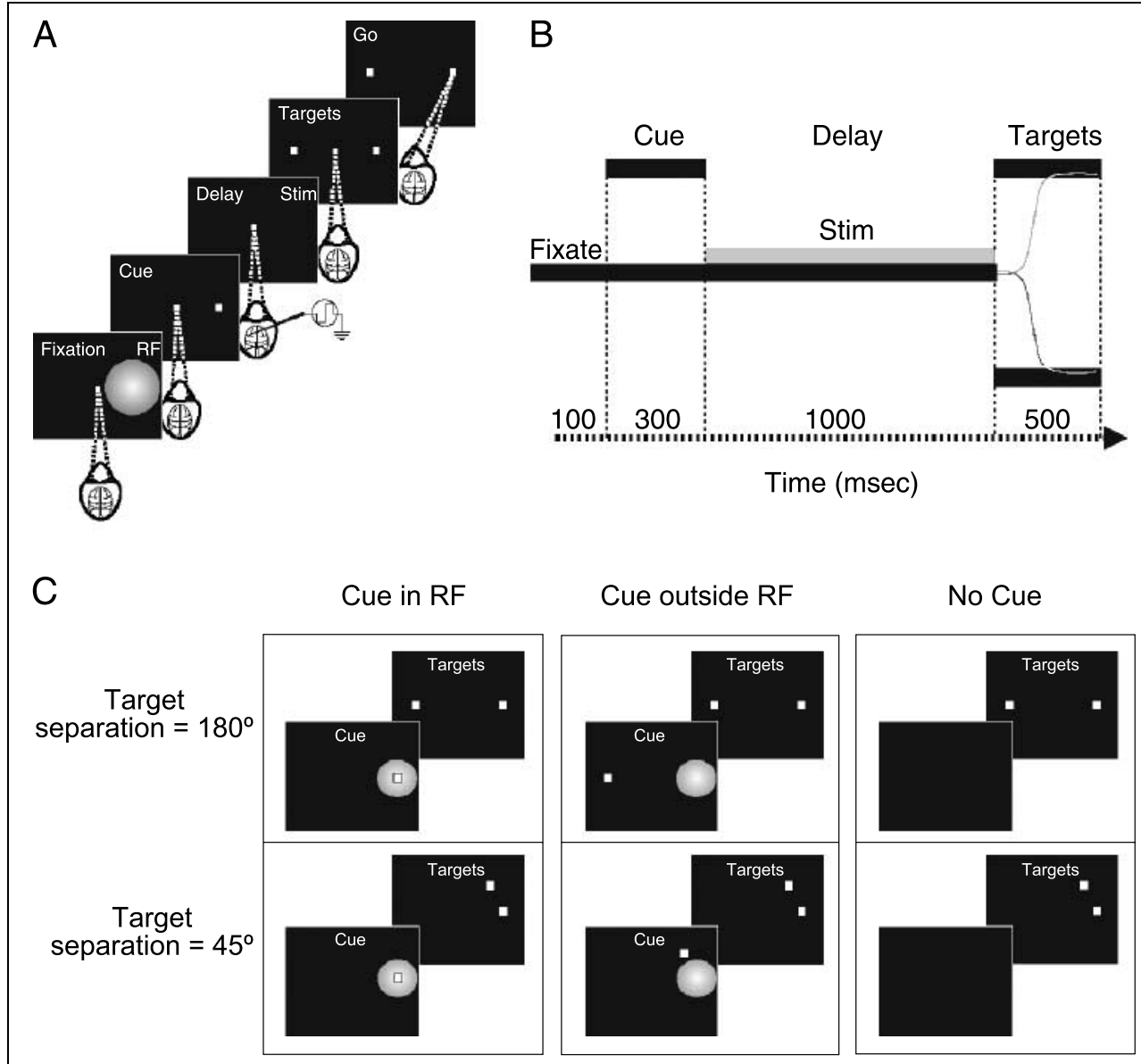

Cue outside RF No Cue
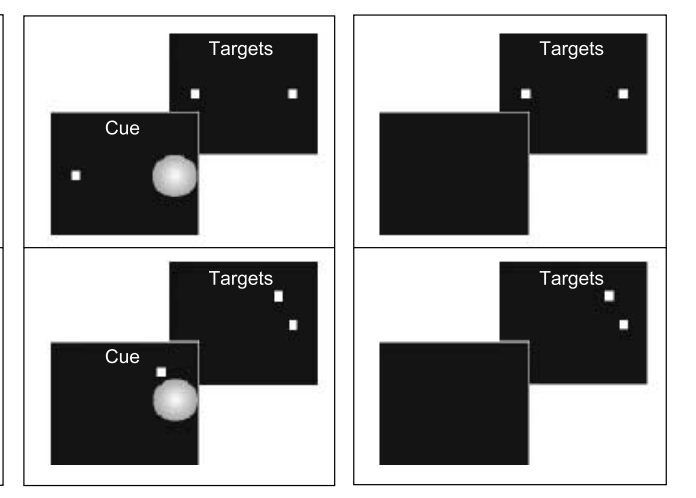
Figure 2. Example of microstimulation effects on saccade target selection: $\mathrm{RF} / \mathrm{MF}$ attraction effect. (A, B): Target-distractor separation was $180^{\circ}$. The FEF site was electrically stimulated with a subthreshold current of $15 \mu \mathrm{A}$ (threshold $=30 \mu \mathrm{A}$ ). Remembered cue location was in the RF/MF (A) and opposite to RF/MF (B). Small dots (black color for stimulated [STIM] and gray color for nonstimulated [NOSTIM] trials) represent eye position samples and larger dots represent the saccade endpoints. The light gray arrow represents the direction and amplitude of the electrically elicited saccades (and was considered the center of site's movement field). (C, D): Target-distractor separation was $45^{\circ}$. Both FEF sites show robust deflection of saccade traces towards cells movement field (dark gray arrow represents the tuning vector during the presaccadic epoch of the MGS task, while the light gray vector has a similar meaning as in $\mathrm{A}$ and $\mathrm{B})$. The sites were electrically stimulated with subthreshold currents of 10 and $40 \mu \mathrm{A}$, respectively (thresholds $=20$ and $80 \mu \mathrm{A}$ ).
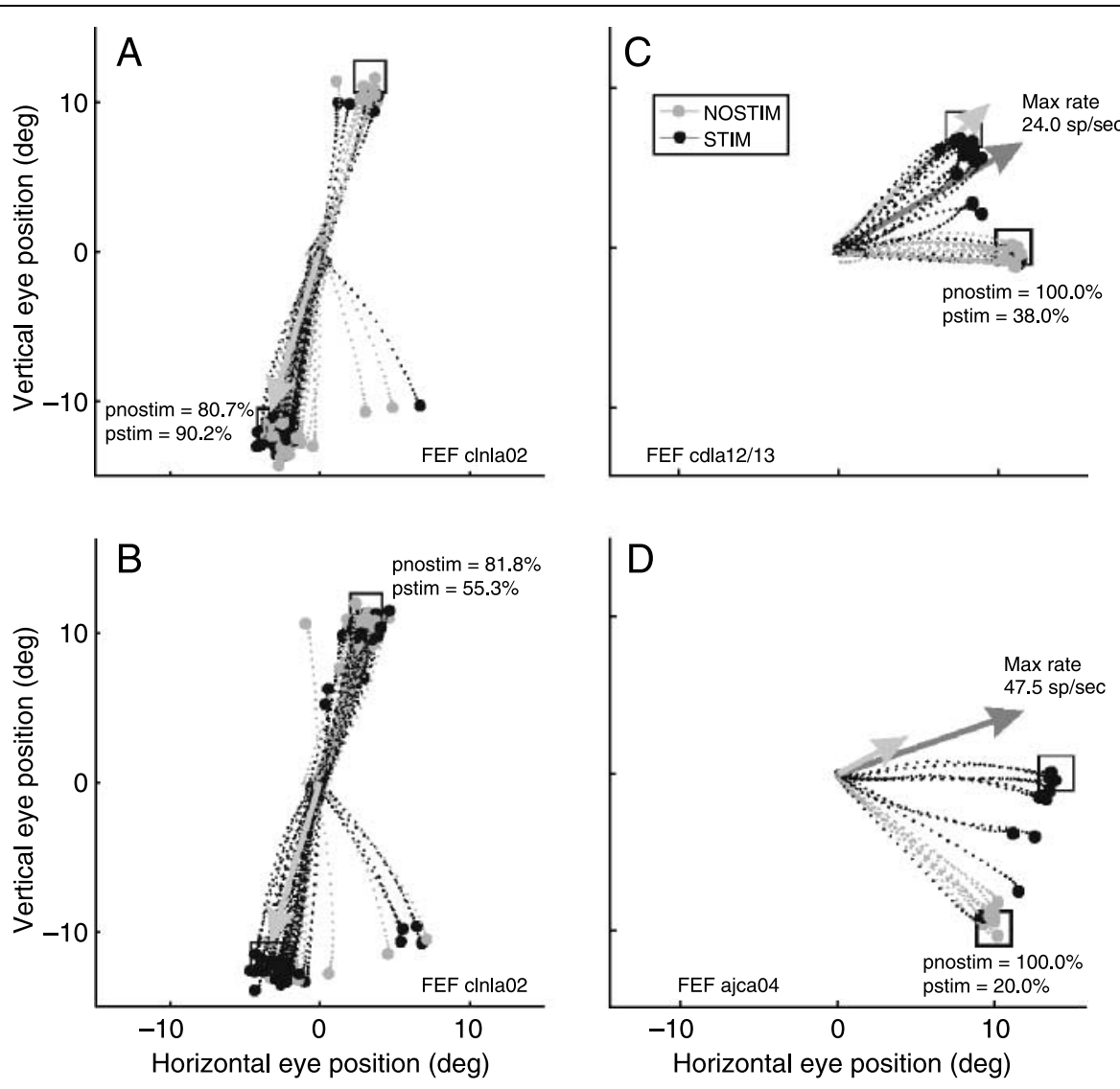

Examples of stimulation effects for the smaller targetdistractor separation are shown for two stimulation sites in Figure $2 \mathrm{C}$ and $\mathrm{D}$. The arrows indicate the electrically evoked saccade vectors (light gray) and neuronal tuning vectors (dark gray). For both sites, stimulation biased saccades toward the MFs, but also increased the number of "averaging" saccades that landed between the target and the distractor. This observation is important in terms of biased competition models of target selection that predict a shift from vector averaging (VA) to winnertake-all (WTA) selection (e.g., Ferrera, 2000).

Stimulation during the delay interval did not always bias saccades toward the receptive/movement field (RF/ $\mathrm{MF})$. At some sites, the monkey consistently chose the target that was outside the RF/MF. An example of this is shown in Figure 3A. The cell that was recorded at this site had visual and delay activity that were tuned for $315^{\circ}$ (lower right quadrant), but no movementrelated activity. The saccade threshold was greater than $100 \mu \mathrm{A}$. The monkey always chose the RF target on non- stimulated trials, but chose the target outside the RF on just over $10 \%$ of the stimulated trials. In another case (Figure 3B), the monkey chose the non-RF target on stimulation trials 8 out of 10 times. This was also a case where the recorded neuron had visual and delay activity without movement-related activity and the stimulation threshold was over $100 \mu \mathrm{A}$. Selection of the non-RF/MF target may reflect the inhibition of a previously cued location, also known as inhibition of return (IOR; Posner, Rafal, et al., 1985).

The main results for all 53 experiments are summarized in Table 1 and Figure 4. None of the analyses described below showed any systematic differences between low-threshold FEF sites and high-threshold sites. Nor did we find systematic differences based on whether cells recorded at the stimulation site were mainly visual or movement-related. We therefore analyzed all 53 experiments as a single group, and we refer to the preferred spatial location of the neurons collectively as the "receptive/movement field" or "RF/MF." 
Figure 3. Example of microstimulation effects on saccade target selection: $\mathrm{RF} / \mathrm{MF}$ repulsion effect. (A) Target-distractor separation was $180^{\circ}$. (B) Target-distractor separation was $45^{\circ}$. Same conventions apply as in Figures 2A-D. The two prefrontal sites were electrically stimulated with subthreshold currents of 40 and $50 \mu \mathrm{A}$, respectively (thresholds $>100 \mu \mathrm{A}$ for both sites).

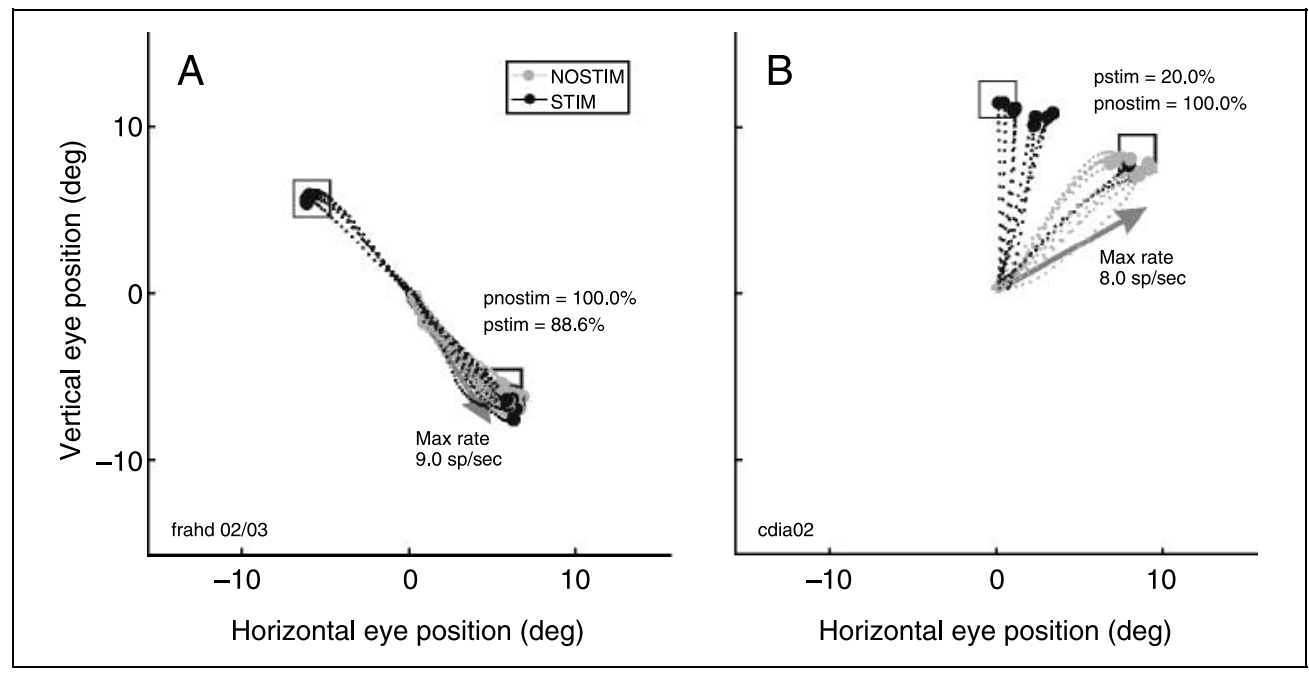

(There were some differences between monkeys. Notably, it was more difficult to obtain significant stimulation effects in Monkey A. This monkey also had much more experience with the task than the other two.) At the population level, two effects stood out. The first is that the stimulation effect depended on the presence of the spatial cue. In the absence of the cue, monkeys were allowed to choose freely and stimulation had no significant effect on the selection of the saccade target. (The designation of the correct choice on no-cue trials was made arbitrarily, hence, the effect of stimulation on percent correct for this condition can be considered an estimate of the bias in the Q-test given the present data.) When the cue was present, more than half the experiments showed significant effects of stimulation on both percent correct choices and the percent of trials on which the monkeys chose the RF/MF stimulus.

The strongest effect of stimulation was to impair behavioral performance (percent correct) on the task (Figure 4A). The reduction in performance was accom-

Table 1. Summary of Stimulation Effects during Delayed Spatial Matching

\begin{tabular}{|c|c|c|c|c|c|}
\hline Outcome & Cue Condition & $\begin{array}{c}\text { Difference (stim - } \\
\text { nostim; mean } \pm S E)\end{array}$ & $t$ test $p$ & $\begin{array}{c}\text { Significant Experiments } \\
(Q-t e s t, p<.05)\end{array}$ & $\begin{array}{c}\text { Significant Experiments } \\
(Q \text {-test, } p<.01)\end{array}$ \\
\hline \multirow[t]{4}{*}{$\%$ correct } & \multirow[t]{4}{*}{ Present } & \multirow[t]{4}{*}{$-7.8 \pm 1.3$} & \multirow[t]{4}{*}{$<10^{-6}$} & $n=35 / 53(65 \%)$ & $n=27 / 53(51 \%)$ \\
\hline & & & & Monkey A: 4/19 & Monkey A: 3/19 \\
\hline & & & & Monkey C: $21 / 23$ & Monkey C: 16/23 \\
\hline & & & & Monkey F: 10/11 & Monkey F: 8/11 \\
\hline \multirow[t]{4}{*}{$\%$ toward $\mathrm{RF} / \mathrm{MF}$} & \multirow[t]{4}{*}{ Present } & \multirow[t]{4}{*}{$2.6 \pm 1.1$} & \multirow[t]{4}{*}{0.017} & $n=31 / 53(57 \%)$ & $n=21 / 53(40 \%)$ \\
\hline & & & & Monkey A: 4/19 & Monkey A: 1/19 \\
\hline & & & & Monkey C: $17 / 23$ & Monkey C: $11 / 23$ \\
\hline & & & & Monkey F: 10/11 & Monkey F: 9/11 \\
\hline \multirow[t]{4}{*}{$\%$ correct } & \multirow[t]{4}{*}{ Absent } & \multirow[t]{4}{*}{$-1.4 \pm 1.4$} & \multirow[t]{4}{*}{0.8} & $n=9 / 53(17 \%)$ & $n=0 / 53(0 \%)$ \\
\hline & & & & Monkey A: 1/19 & \\
\hline & & & & Monkey C: 6/23 & \\
\hline & & & & Monkey F: 2/11 & \\
\hline \multirow[t]{4}{*}{ \% toward $\mathrm{RF} / \mathrm{MF}$} & \multirow[t]{4}{*}{ Absent } & \multirow[t]{4}{*}{$-0.6 \pm 1.6$} & \multirow[t]{4}{*}{0.35} & $n=13 / 53(24 \%)$ & $n=0 / 53(0 \%)$ \\
\hline & & & & Monkey A: 1/19 & \\
\hline & & & & Monkey C: $10 / 23$ & \\
\hline & & & & Monkey F: 2/11 & \\
\hline
\end{tabular}


Figure 4. Scatterplots (stim vs. no-stim) for percent correct performance (A) and percent choices toward the RF/MF (B). Dashed lines indicate the null hypothesis: stim $=$ no-stim. Note that the axes in $\mathrm{A}$ and $\mathrm{B}$ have the same scale but different ranges. The 50\% performance/choice level is indicated by dotted lines in $\mathrm{B}$.
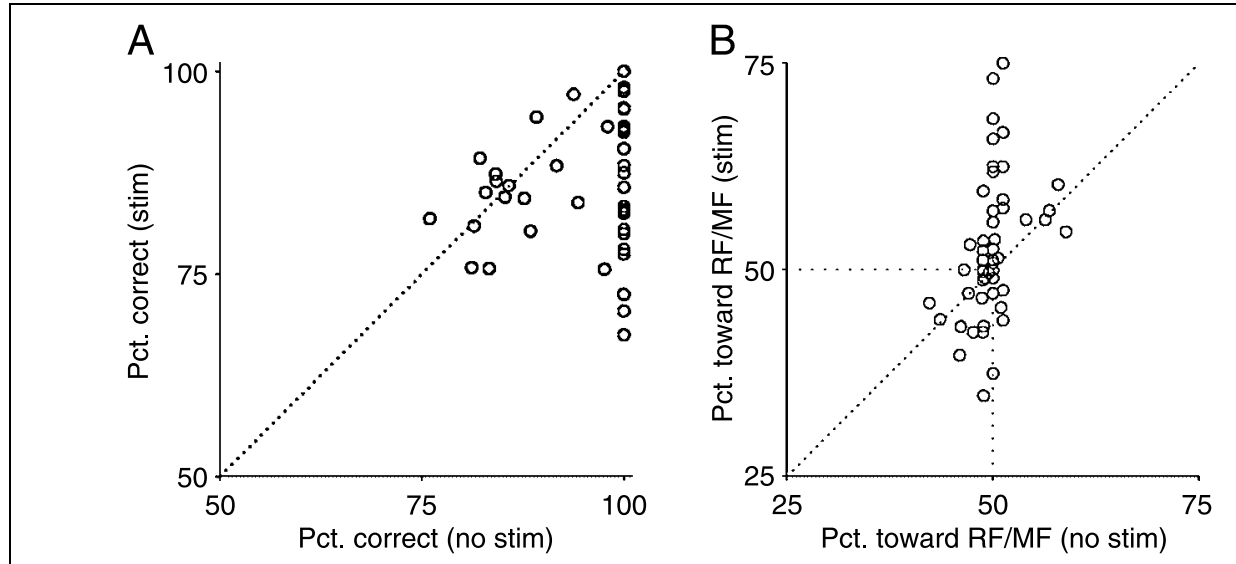

panied by a subtler shift in the proportion of saccades directed toward the RF/MF of the site (Figure 4B). (Note that the Q-test only indicates whether there was a difference in the choices made on stimulated vs. nonstimulated trials, it does not indicate whether those choices were toward or away from the RF/MF.) Monkeys chose the stimulus in the RF/MF more frequently on stimulation trials in 34/53 (64\%) of experiments and 23 (43\%) of these were significant (Q-test, $p<.05$ ). Monkeys chose the stimulus outside the RF/MF more frequently on stimulation trials in $15 / 53$ (28\%) of experiments, and $8(15 \%)$ were significant. There were no significant differences between monkeys either in terms of change in percent correct (stim - no-stim) or change in percentage of choices toward the RF/MF (one-way ANOVA, factor $=$ monkey; $p>.05$, Bonferroni corrected).

Stimulation also resulted in a broadening of the distribution for the proportion of saccades directed toward the RF/MF (Figure $4 \mathrm{~B}$ ). This broadening was not simply a side effect of the decrease in percent correct. Any systematic bias in the monkey's behavior relative to the preferred location of the stimulation site will necessarily result in decreased performance. However, it is possible to have a significant reduction in percent correct without a systematic effect on $\mathrm{RF} / \mathrm{MF}$ versus non-RF/MF choices, if stimulation simply caused the monkey to behave more randomly. In this case, one would expect the saccades made toward the RF/MF to remain near $50 \%$. For stimulation to cause the distribution to spread away from $50 \% \mathrm{RF} / \mathrm{MF}$ choices, as in Figure $4 \mathrm{~B}$, the choices at each site must be systematically biased toward or away from the RF/MF.

If stimulation merely caused an increase in random behavior, one would further predict that the targetdistractor and RF/MF-non-RF/MF effects would be independent. In fact, they were not. Nearly every site that showed a significant effect (Q-test, $p<.05$ ) for targetdistractor (percent correct) also showed an effect for RF-non-RF $(n=28 / 35)$. Further evidence that the effects are related was found in the correlation of the Q-test $p$ values. The logs of the $p$ values for the effect of stimulation on percent correct and percent $\mathrm{RF} / \mathrm{MF}$ were found to be highly correlated $(R=.78)$. These results suggest that prefrontal stimulation had a systematic rather than random effect on saccade choices.

The effects of stimulation depended on the spatial proximity of the target and distractor. When the targetdistractor separation was $45^{\circ}$ ( $7^{\circ}$ of visual angle), there was a significant difference in percent correct between stimulated and nonstimulated trials for 28/53 (53\%; Qtest, $p<.05$ ) experiments and the mean difference (stim - no-stim) was $-10.4 \%\left(p<10^{-6}\right.$; paired $t$ test). For the separation of $180^{\circ}$ ( $20^{\circ}$ of visual angle), the difference in performance was significant for 19/53 (36\%) experiments and the mean was $-5.6 \%\left(p<10^{-5}\right)$. The difference in $\mathrm{RF} / \mathrm{MF}$ choices at $45^{\circ}$ separation was significant for $27 / 53$ (51\%) experiments and the mean bias toward the RF/MF was $3.6 \%(p<.05)$, while for $180^{\circ}$, the difference was significant for $20 / 53$ (38\%) experiments and the mean bias was $1.4 \%(p=.22)$. Thus, all effects of stimulation were significantly stronger for the smaller target-distractor separation. Excluding saccades with latencies $<100 \mathrm{msec}$ slightly increased the magnitude of all stimulation effects.

To avoid punishing the monkey for choosing the distractor, stimulated trials were always rewarded, and nonstimulated trials were rewarded only when performed correctly. One could therefore argue that microstimulation did not have a direct affect on the monkey's choices, but simply served as a signal that he could relax his performance. If the monkey was able to discriminate stimulated from nonstimulated trials (perhaps by feeling a "tingle" during stimulation, or seeing flashes of light), then he may simply have chosen to act randomly on stimulated trials. To address this, we ran 12 experiments using a modified task in which the monkey was required to perform correctly on both stimulated and nonstimulated trials. The comparison between performance on the original and modified tasks was as follows: $89.0 \%$ versus $85.2 \%$ correct in stimulated trials ( $p=.17 ; t$ test $)$ and $98.8 \%$ versus $84.8 \%$ in nonstimulated trials $\left(p<10^{-14} ; t\right.$ test $)$. 
Stimulation on cued trials significantly $(p<.05$; Q-test $)$ biased the monkey's choices toward the RF in 8/12 experiments and away from the RF in 3/12 experiments. It is noteworthy that performance in the modified task was worse for both stimulated and nonstimulated trials. If monkeys could discriminate between stimulated and nonstimulated trials, performance during stimulated trials should have been better for the modified task than the original task. It should be noted that enforcing correct behavior on stimulated trials may effectively result in punishment for behavior that the monkey "believes" was correct. The resulting confusion or frustration may account for the drop in performance on nonstimulated trials.

\section{Theoretical Implications}

Some models of two-choice decision processes make explicit predictions about the relationship between response speed and accuracy. Models that accumulate or integrate evidence over time predict that errors should decrease as response latency increases (Ditterich et al., 2003; Ratcliff, 2002; Usher \& McClelland, 2001). To investigate this, we separated the data into trials in which the choice saccade was within $\pm 2^{\circ}$ of the target ("accurate" trials) and those in which it was directed elsewhere ("inaccurate" trials). There were a total of $n=6494$ (stimulation trials) and $n=6157$ (nonstimulated trials) choice saccades used for this analysis. Saccades with latencies less than $100 \mathrm{msec}$ were not included. This criterion excluded 188 (2.9\%) and 196 (3.2\%) stimulated and nonstimulated trials, respectively. We found evidence for an inverse speed-accuracy tradeoff (Figure 5A) and for an interaction between the spatial cue and stimulation (Figure 5B). In general, latencies for accurate saccades were fairly short; $157 \pm 24$ (mean \pm $S D)$ msec for the no-cue condition regardless of whether stimulation was applied versus $154 \pm 25 \mathrm{msec}$ for the cued condition without stimulation and $157 \pm 27 \mathrm{msec}$ for the cued condition with stimulation (Figure 5A). The effect of stimulation was not significant in the no-cue condition (stimulated vs. nonstimulated saccade latency, paired $t$ test, $p=.7$ ), but was significant in the cued condition $\left(p<10^{-7}\right)$ (Figure $5 \mathrm{~B}$ ). The difference between the cue and no-cue means (Figure 5B) was also significant ( $t^{\prime}$ test for distributions with unequal variance and number of observations, $p=.003)$. For inaccurate saccades, the mean latencies were $170.6 \pm 27 \mathrm{msec}$ (nonstimulated trials) and $170.1 \pm 32 \mathrm{msec}$ (stimulated trials), significantly higher than the corresponding latencies for accurate saccades $\left(p<10^{-10}\right.$ for both stimulated and nonstimulated trials; $t^{\prime}$ test). There was no relationship between latency and accuracy for uncued trials. These results do not support the idea that performance on this task is limited by the accumulation of evidence over time (as one would expect given that there was no speeded reaction time component to the task). However, they do suggest that stimulation interferes in saccade target selection when memory of the cue location and a decision are required but not when the monkey is free to choose either target.

Target selection for some voluntary eye movements (i.e., smooth pursuit) has been characterized as a transition from VA (Lisberger \& Ferrera, 1997) to WTA pursuit, and simulations of competitive network models have shown that a single network can operate in both VA and WTA modes (Ferrera, 2000). If a similar mechanism exists for saccade target selection, then there
Figure 5. (A) Mean latency plot. The circle and dot represent the no-cue trials whereas the empty and filled squares represent the cued trials. Empty symbols represent the nonstimulation condition and the dot and filled square represent the stimulation condition. (B) Distribution of latency difference between stimulation and nonstimulation trials for cued and uncued trials. Filled bars represent the cued trials whereas the empty bars represent the uncued trials. The data were split by target-distractor separation so that each stimulation experiment contributes two observations to each distribution (cued and noncued).

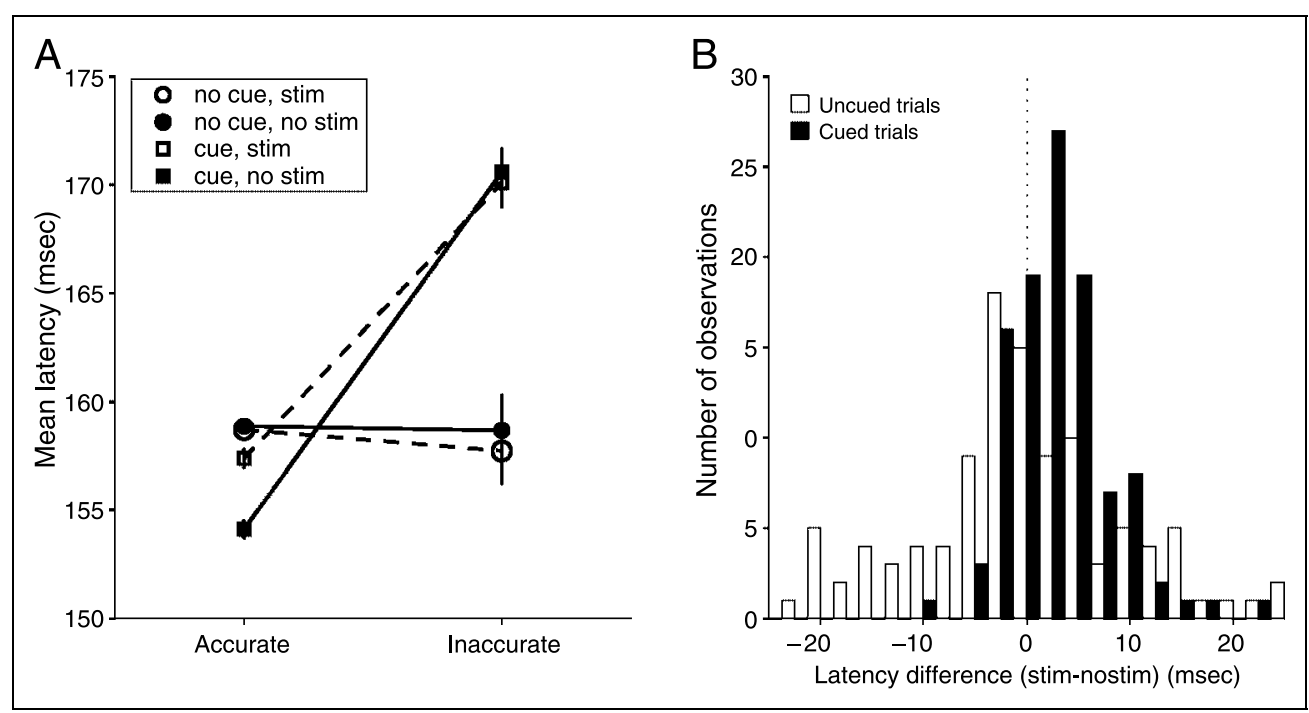


should be an effect of stimulation on the production of averaging saccades. To quantify saccade averaging, on each trial, the saccade vector $R$ is expressed as a weighted summation of the visual target $(T)$ and distractor $(D)$ vectors (see Data Analysis in Methods). We analyzed VA saccades for $45^{\circ}$ and $180^{\circ}$ target separations using only cued trials. The results are shown in Figure $6 \mathrm{~A}$ and $\mathrm{B}$. Saccades for trials with angular separation $=45^{\circ}$ were analyzed using a two-dimensional vector decomposition $\left(R=w_{\mathrm{t}}{ }^{*} T+w_{\mathrm{d}} * D\right)$. The tips of the basis vectors $(T, D)$ were the target and distractor positions. For the $180^{\circ}$ target-distractor separation, the two-dimensional vector analysis is invalid (because the basis vectors are co-linear), therefore we used a onedimensional analysis $\left(R=w^{*} T+[1-w]^{*} D\right)$ (Groh, Born, \& Newsome, 1997). For both the one- and twodimensional analyses, the weights can be calculated directly from the data, and it is not necessary to fit regression functions (as in Groh et al., 1997). The onedimensional model assumes that the weights fall along the line $w_{\mathrm{d}}=1-w_{\mathrm{t}}$. Figure $6 \mathrm{C}$ validates this assumption for the $45^{\circ}$ separation and we assume that it holds for the $180^{\circ}$ separation as well. Figure $6 \mathrm{C}$ also shows that the target and distractor weights were strongly negatively correlated $(R=-.92$ for nonstimulated trials, $R=-.88$ for stimulated trials). Hence, it is only necessary to show the distributions of target weights (Figure 6A and B). These distributions reveal that the percentage of averaging saccades was small. However, for the $45^{\circ}$ target-distractor separation, there were more averaging saccades and more saccades directed toward the distractor on stimulated trials compared with nonstimulated trials. Figure $6 \mathrm{D}$ shows the percentages of averaging saccades, defined as those for which the absolute value of $\left(w_{\mathrm{t}}-w_{\mathrm{d}}\right)<0.3$. For the $45^{\circ}$ separation, there were significantly more averaging saccades on stimulated versus nonstimulated trials $(p=.0025$, unpaired $t$ test). For the $180^{\circ}$ separation, there were very few averaging saccades in either condition, and the difference was not significant ( $p=.25$, unpaired $t$ test).

\section{DISCUSSION}

We found that in majority of the experiments, prefrontal (periarcuate) microstimulation significantly biased saccade choices even though there was little or no un-
Figure 6. Saccade vector analysis. (A, B) Distribution of saccade proportion as a function of target weight for target separation $=$ $45^{\circ}$ (A) and for target separation $=180^{\circ}(\mathrm{B})$. (C) Regression plot of distractor versus target weight for $45^{\circ}$ separation trials. (D) Percentage of saccade averaging trials as a function of target separation.

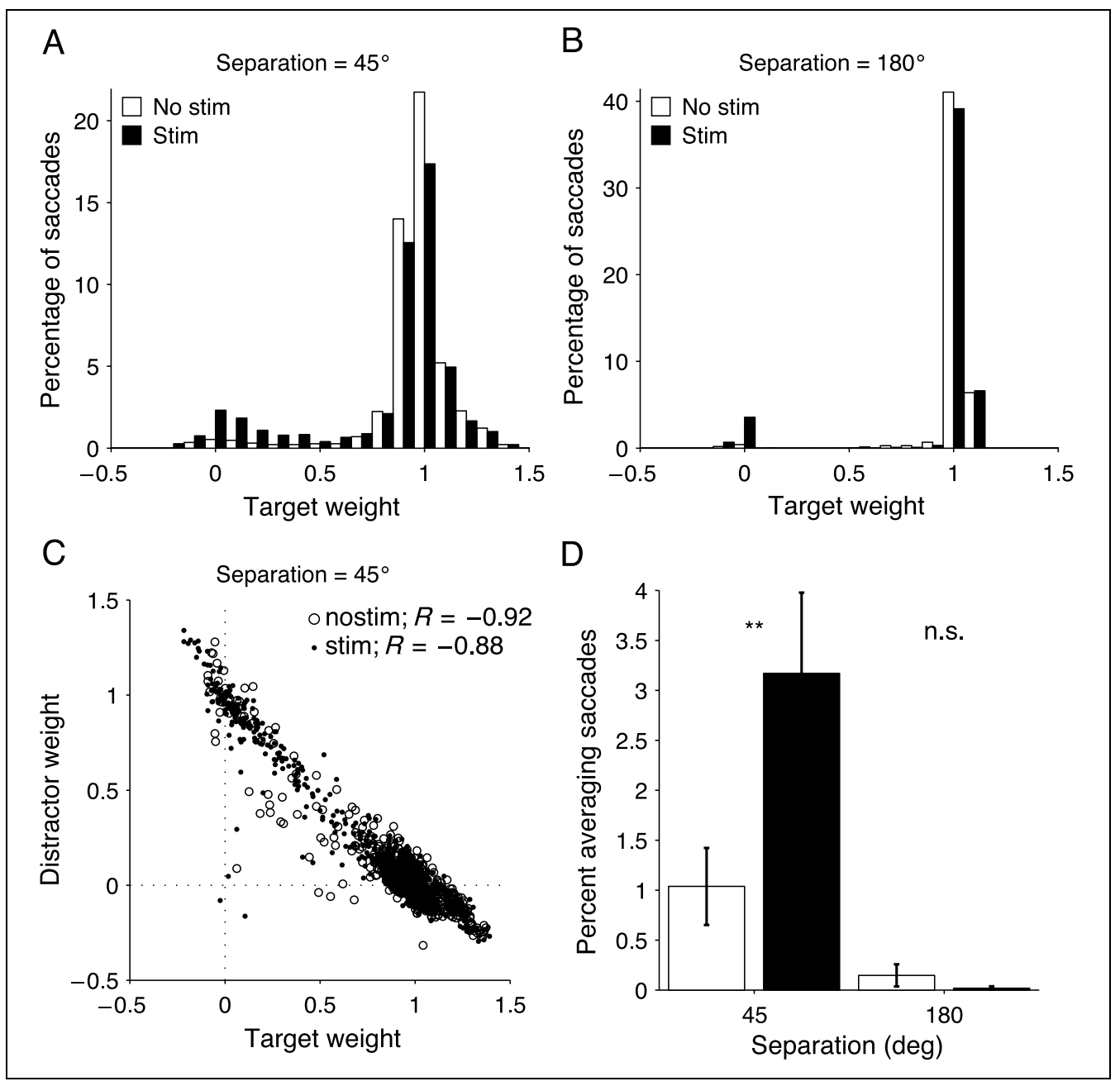


certainty about the correct (rewarded) response. Stimulation resulted in a decrease in task performance (percent correct) and also a small increase in the proportion of saccades directed toward the $\mathrm{RF} / \mathrm{MF}$ of the stimulation site. The overall strength of the effect of stimulation on saccade choices was mitigated by the fact that there were statistically significant effects in both directions; of 31 experiments showing a significant effect of stimulation, 23 had a bias toward the stimulus inside the RF/MF and 8 had a bias toward the non-RF/ MF stimulus. Stimulation also resulted in a small increase in saccade latency, but only for cued trials, that is, those trials for which the monkey was required to remember the cue and make a decision. Stimulationrelated changes in behavioral performance were significantly stronger when the target and the distractor were in close proximity ( $7^{\circ}$ of visual angle) than when they were diametrically opposed. Furthermore, for the smaller target-distractor separation, stimulation increased the percentage of averaging saccades by a factor of 3. We found no significant differences between low- and high-threshold sites for any stimulation effect.

Before one can draw any conclusions from these experiments regarding the role of the prefrontal cortex in oculomotor decision-making, several competing hypotheses must be ruled out. One of these is the "blow to the head" theory, which posits that stimulation affects performance simply by distracting the monkey, as if each stimulation trial was accompanied by a noxious stimulus. In this case, one would expect the monkey to behave randomly on stimulation trials, resulting in a decrease in overall performance, as observed. However, one would not necessarily expect any systematic bias in choosing the stimulus inside the RF/MF. The fact that such a bias was found in 31 of the 34 experiments that showed a significant decrease in performance suggests that stimulation altered performance in a systematic rather than random manner. A related idea is that stimulation causes some sensation ("tingle") that acts as a signal effectively reducing the monkey's motivation as stimulation trials were always rewarded. To address this, experiments were run in which stimulation trials were rewarded only when completed correctly. This should have restored the monkeys' motivation and increased their percent correct. In fact, there was a slight decrease in performance when the reward contingency was enforced on stimulation trials.

Another possible explanation for the results is the "phosphene" theory, which supposes that electrical stimulation results in a spatially localized visual percept. This percept could compete with the memory of the visual cue and thereby cause monkeys to choose the RF/ MF stimulus more frequently. To control for this, each experiment contained a set of randomly interleaved trials which had no visual cue and monkeys were free to choose either stimulus. If the phosphene theory was correct, then monkeys should choose the RF/MF stim- ulus more frequently in the no-cue condition relative to the cued condition. In fact, in the absence of the cue, there was no significant effect of stimulation on either percent correct or percent RF/MF choices (see Table 1). A similar pattern of results was seen in the saccade latency data. Stimulation trials had longer choice saccade latencies than nonstimulated trials but only for cued trials. Thus, for percent correct, percent RF/MF choices, and saccade latency, effects were found only when the cue was present, suggesting that the electrical stimulus cannot take the place of the visual cue. It is therefore likely that in these experiments stimulation in the prefrontal cortex exerted its effects at a postsensory stage of processing.

Attention and oculomotor target selection have been modeled as a biased competition between alternative stimuli or responses (Ferrera, 2000; Reynolds, Chelazzi, \& Desimone, 1999; Ferrera \& Lisberger, 1995). Sustained activity in the prefrontal cortex may represent a top-down attentional bias that influences the outcome of this competition (Moore \& Armstrong, 2003; Kim \& Shadlen, 1999). There is also evidence for competitive interactions within the FEF itself (Burman \& Bruce, 1997). One characteristic of a competitive network is a gradual shift from VA to WTA behavior as the strength of the bias increases. If electrical stimulation reduces or competes with the bias derived from the visual cue, then it should not only shift responses toward the $\mathrm{RF} / \mathrm{MF}$, but should also increase the proportion of averaging saccades. This expectation is confirmed by the data in Figure 6. Another effect of competition is to increase response latency. The data in Figure 5B are consistent with the idea that prefrontal stimulation weakens the bias associated with the visual cue and thereby increases the amount of time needed for the decision network to reach a stable state (Wang, 2002).

The effects observed during electrical stimulation of the prefrontal cortex are substantially weaker than those found by stimulating visual area MT (Nichols \& Newsome, 2002; Bisley, Zaksas, \& Pasternak, 2001; Groh et al., 1997; Salzman \& Newsome, 1994). There are several likely reasons for this. MT may be uniquely critical for the processing of visual motion, as evidenced by lesion studies (Lauwers, Saunders, Vogels, Vandenbussche, \& Orban, 2000; Pasternak \& Merigan, 1994; Newsome \& Pare, 1988). The prefrontal cortex, including the FEFs, is probably only one node of a distributed network for saccades (Schiller \& Chou, 1998). Furthermore, timing is critical for stimulation effects (Opris, Barborica, \& Ferrera, 2001; Seideman et al., 1998). Many studies showing strong stimulation effects have applied the electrical stimulus coincident with the visual stimulus or movement. Terminating the stimulus as late as 40-60 msec before the movement can result in no effect whatsoever (Glimcher \& Sparks, 1993). In the present study, the electrical stimulus was terminated a mini- 
mum of 100 msec before the movement (except for the roughly $3 \%$ of trials that had latencies shorter than $100 \mathrm{msec}$; however, none of the stimulation effects was driven by short latency saccades, in fact, all effects were marginally stronger when such saccades were excluded). It should be noted that Bisley et al. (2001) found that stimulation in MT during the delay interval of a memory-for-motion task resulted in a general impairment in performance, comparable in magnitude to the effects found in the present study. However, they found that stimulation during the cue interval had more powerful and more selective effects.

A study similar to the current one was performed by Carrello and Krauzlis (2004) who applied subthreshold stimulation in the intermediate layers of the superior colliculus (SC) while monkeys performed a saccade or smooth pursuit target selection task. They showed that SC stimulation biases target selection toward the contralateral visual field, a result that is consistent with our findings in the prefrontal cortex. However, Carrello and Krauzlis applied stimulation before and during the choice saccade, whereas in the present study, stimulation ended at least $100 \mathrm{msec}$ before saccade onset. This may account for differences in the magnitude of the stimulation effect between the two studies, that is, the effects appear to be somewhat larger in the Carrello and Krauzlis study. There are also important differences in the behavioral tasks used in the two studies. Carrello and Krauzlis cued the color of the rewarded target, whereas in the current study, we cued target location. We have trained monkeys on both color- and locationcue tasks (Ferrera et al., 1999) and have observed anecdotally that they perform much better on the location-cue task (unpublished observations). More importantly, FEF neurons respond very differently on delayed version of these tasks (Ferrera et al., 1999). For the color-cue task, FEF neurons show no cue-dependent delay activity and have a weak to moderate difference in presaccadic firing that favors the target within their RF. For the location task, FEF neurons show robust delay activity and a strong selection bias in their presaccadic activity. Hence, for the location task, stimulation delivered during the delay period may interact with delay period activity within the FEF itself. For the color task, the FEF is probably reading the output of colorselective delay activity in other regions such that delay period stimulation in FEF would be expected to have little effect on behavior.

Moore and Fallah (2001, 2004) have shown that subthreshold stimulation in FEF can enhance the detection of a subtle change in a visual target, an effect consistent with orienting of visual attention toward the RF/ MF of the stimulation site. Spatial orienting of attention was likely engaged in the present study. However, our task was deliberately designed using bright, highcontrast targets, so it is unlikely that limited processing capacity played a large role in the animals' performance.
Our goal was, in fact, to eliminate the role of perceptual detectability or discriminability and thereby show a direct effect on response selection. We feel that our results, together with those of Moore and Fallah, reinforce the linkage between spatial attention and response selection.

The net systematic effect of prefrontal microstimulation in the current study was mitigated by the fact that there were effects in opposing directions; although stimulation generally biased responses toward the RF/ $\mathrm{MF}$, there were also sites that showed significant bias toward the non-RF/MF stimulus. This observation is not unprecedented. Groh et al. (1997) similarly found that stimulation in MT caused shifts in smooth pursuit eye velocity in the null direction in a substantial percentage (30-40\%) of their experiments. They were subsequently able to explain their null-direction effects based on center/surround organization in MT (Born et al., 2000). Null direction effects (i.e., increase in non-RF/MF choices) for spatial tasks, such as that used in the present experiments, may be related to the inhibition of a previously cued location, known as "inhibition of return" (IOR), which provides a bias in favor of novel locations (Posner \& Cohen, 1984). The present results suggest that the dlPFC (including the FEF) may be involved in IOR generation. Other studies indicate that IOR is closely related to the eye movement system (Bichot \& Schall, 2002).

In conclusion, we found that prefrontal microstimulation biased saccade target selection. Generally, the bias was toward the preferred location of the stimulation site, but occasionally, there were biases away from the preferred location. This bias was unlikely to stem from an interaction of the electrical stimulation with sensory evidence in such a way that the location of the perceived stimulus used to cue the target selection was altered. Rather, the data are more consistent with the idea that prefrontal stimulation introduces a selection bias that directly affects the competition between alternative responses.

\section{METHODS}

Experiments were performed on three male (8, 6, and $5 \mathrm{~kg}$ weight) rhesus monkeys (Macaca mulatta). The treatment of the monkeys was in accordance with the guidelines set by the U.S. Department of Health and Human Services (NIH) for the care and use of laboratory animals.

\section{Behavioral Paradigm}

We trained monkeys to make saccades to the remembered location of the visual cue in an MGS task with eight target positions, equally spaced $\left(45^{\circ}\right)$ around the clock (Opris et al., submitted; Funahashi et al., 1989). We then electrically stimulated the same sites in a spatial MTS 
saccade task (Figure 1). In the MTS task, at the beginning of each trial, the monkey fixated a small white square. A peripheral cue was flashed for $300 \mathrm{msec}$. Spatial cues were located either within the RF or at a location outside the RF (non-RF) with equal probability. The fixation point was present and the animal was required to maintain fixation during the entire delay epoch. At the end of the delay interval, the fixation spot disappeared and two targets were presented for $500 \mathrm{msec}$. Monkeys were then allowed to make a saccade to one of the targets. A critical feature of the MTS task was the angular separation between the matching target and the distractor. In cued trials, the distractor was separated from the target by either $180^{\circ}$ (Figure $1 \mathrm{~A}$ ) or $45^{\circ}$ (Figure 1B). In the no-cue trials, the monkey was free to choose either target (both targets were rewarded).

During each experiment, neuronal activity was first conducted to determine the $\mathrm{RF} / \mathrm{MF}$ (i.e., the part of visual space that neurons around the electrode tip responded to). The target matching the remembered cue location was programmed to be within the RF/MF in 50\% of trials and outside the $\mathrm{RF} / \mathrm{MF}$ in $50 \%$ of trials. To estimate the preferred location, we used the visual, delay, or movement epoch activity recorded during an MGS task. When low-threshold FEF sites were encountered, the MF was also estimated by the vector of the electrically elicited saccades. Thresholds for electrically evoked saccades were determined by varying the stimulation current $(\max 100 \mu \mathrm{A})$ while the monkey performed a fixation task (Opris et al., 2001). This procedure was used to classify stimulation sites as being within the low-threshold FEF (threshold $\leq 50 \mu \mathrm{A}$ ) or at nearby locations in the anterior bank of the arcuate sulcus (Burman \& Bruce, 1997; Bruce \& Goldberg, 1985; Bruce, Goldberg, Bushnell, \& Stanton, 1985). The stimulation current for the spatial MTS task was set to the lesser of half the electrically evoked saccade threshold or $50 \mu \mathrm{A}$, to ensure that application of current would not by itself generate a response (Moore \& Armstrong, 2003; Burman \& Bruce, 1997; Groh et al., 1997). Subthreshold stimulation was applied during the entire delay interval while the fixation point was present. The stimulation ended at the same time the fixation point was turned off. Stimulation and nonstimulation trials were randomly interleaved. On nonstimulated trials, monkeys were rewarded for choosing the target that matched the cue location. On stimulated trials, monkeys were rewarded for choosing either target.

\section{Neuronal Recording and Stimulation}

An MGS task with eight target locations was used to determine the preferred location of neurons at each stimulation site. Action potentials were discriminated from background noise using a time-amplitude window. The spikes were time-stamped with a resolution of $0.1 \mathrm{msec}$. Eye position was digitized at $1 \mathrm{kHz}$ with 12-bit resolution and stored together with the spike trains. The preferred location was computed as a weighted average of the mean firing rate for each cue location (center-of-mass vector). Sites were stimulated through the same electrode used to record neuronal activity. The stimulation consisted of a train of 0.2 msec biphasic pulses at a rate of 350 pulses $/ \mathrm{sec}$ and was delivered by an optically isolated pulse stimulator (AM Systems, Seattle, WA).

\section{Behavioral Responses}

For the spatial MTS task, the choice saccade was defined as the first saccade initiated after the end of the delay period. The difference between the location of the two choice stimuli and the endpoint of the choice saccade were computed and the chosen stimulus was considered to be the one with the smaller saccade error. This analysis may include a small percentage of stray and averaging saccades. Alternate analyses were performed using eye position windows of different sizes, but this did not substantially alter the results. Performance was quantified as percent correct and percentage of choices made to the $\mathrm{RF} / \mathrm{MF}$ stimulus. To assess the statistical significance of behavioral choice, we performed Cochran's Qtest for dichotomous outcomes. Choice saccade onset was determined using an acceleration criterion (eye acceleration $\geq 500^{\circ} / \mathrm{sec}^{2}$ ). Saccade latency for the MTS task was measured relative to the disappearance of the fixation target at the end of the delay interval.

For saccade vector analysis, we used a one- or twodimensional vector decomposition (Ferrera, 2000; Groh et al., 1997; Lisberger \& Ferrera, 1997). The two basis vectors were the target and distractor locations relative to the center of the display. The "target" was defined as the stimulus matching the cue location and the nonmatching stimulus was the "distractor." It was then possible to express the saccade vector $(R)$ for each stimulation trial as a weighted summation (Lisberger \& Ferrera, 1997) of the component vectors $(T, D)$ :

$$
R=w_{\mathrm{t}} T+w_{\mathrm{d}} D
$$

This was simplified to a one-dimensional analysis when the $T$ and $D$ vectors are collinear by setting $w_{\mathrm{t}}=$ $g$ and $w_{\mathrm{d}}=1-g$ (Groh et al., 1997). This vector decomposition allowed us to identify several interesting outcomes according to the weight distributions. Pure VA corresponds to the case $g=0.5$ (Figure 6A). If the response during stimulation is identical to the response without stimulation $(g=0.0)$, then the outcome is said to be "WTA" for matching target (WTA match). However, if the stimulation overrides the visual target and produces a response into the MF of the stimulation site ( $g=1.0$ ), then the outcome is said to be "WTA" for the nonmatching target (WTA distractor). 


\section{Acknowledgments}

We thank Drs. William Newsome, Michael Shadlen, Michael Goldberg, Christos Constantinidis, and Emilio Salinas for helpful discussion and comments on the manuscript. Technical assistance was provided by Andrea Rocca and Jean Willi. This study was supported by MH15174, MH59244, EJLB, and the James S. McDonnell Foundation.

Reprint request should be sent to Ioan Opris, Center for Neurobiology and Behavior and Department of Psychiatry, Columbia University, New York, NY 10032, USA, or via e-mail: iopris@wfubmc.edu.

\section{REFERENCES}

Baddeley, A. (2003). Working memory: Looking back and looking forward. Nature Reviews: Neuroscience, 4, 829-839.

Barraclough, D. J., Conroy, M. L., \& Lee, D. (2004). Prefrontal cortex and decision making in a mixed-strategy game. Nature Neuroscience, 4, 404-410.

Bichot, N. P., \& Schall, J. D. (2002). Priming in macaque frontal cortex during popout visual search: Feature-based facilitation and location-based inhibition of return. Journal of Neuroscience, 22, 4675-4685.

Bisley, J. W., Zaksas, D., \& Pasternak, T. (2001) Microstimulation of cortical area MT affects performance on a visual working memory task. Journal of Neurophysiology, 85, 187-196.

Born, R. T., Groh, J. M., Zhao, R., \& Lukasewycz, S. J. (2000). Segregation of object and background motion in visual area MT: Effects of microstimulation on eye movements. Neuron, 26, 725-734.

Bruce, C. J., \& Goldberg, M. E. (1985). Primate frontal eye fields: I. Single neurons discharging before saccades.

Journal of Neurophysiology, 53, 603-635.

Bruce, C. J., Goldberg, M. E., Bushnell, M. C., \& Stanton, G. B. (1985). Primate frontal eye fields: II. Physiological and anatomical correlates of electrically evoked eye movements. Journal of Neurophysiology, 53, 714-734.

Burman, D. D., \& Bruce, C. J. (1997). Suppression of task-related saccades by electrical stimulation in the primate's frontal eye field. Journal of Neurophysiology, 77, 2252-2267.

Carrello, C. D., \& Krauzlis, R. J. (2004). Manipulating intent: Evidence for a causal role of the superior colliculus in target selection. Neuron, 43, 575-583.

Cohen, M. R., \& Newsome, W. T. (2004). What electrical microstimulation has revealed about the neural basis of cognition. Current Opinion in Neurobiology, 14, 169-177.

Constantinidis, C., Franowicz, M. N., \& Goldman-Rakic, P. S. (2001). The sensory nature of mnemonic representation in the primate prefrontal cortex. Nature Neuroscience, 5, 175-180.

Courtney, S. M., Ungerleider, L. G., Keil, K., \& Haxby, J. V. (1997). Transient and sustained activity in a distributed neural system for human working memory. Nature, 386, 608-611.

Ditterich, J., Mazurek, M. E., \& Shadlen, M. E. (2003). Microstimulation of visual cortex affects the speed of perceptual decisions. Nature Neuroscience, 6, 891-898.

Eveling, S., Tinsley, C. J., Gaffan, D., \& Duncan, J. (2002). Filtering of neural signals by focused attention in the monkey prefrontal cortex. Nature Neuroscience, 5 , 671-676.
Ferrera, V. P. (2000). Task-dependent modulation of the sensorimotor transformation for smooth pursuit eye movements. Journal of Neurophysiology, 84, 2725-2738.

Ferrera, V. P., Cohen, J. K., \& Lee, B. B. (1999). Activity of prefrontal neurons during location and color delayed matching tasks. NeuroReport, 10, 1315-1322.

Ferrera, V. P., \& Lisberger, S. G. (1995). Attention and target selection for smooth pursuit eye movements. Journal of Neuroscience, 15, 7472-7484.

Funahashi, S., Bruce, C. J., \& Goldman-Rakic, P. S. (1989). Mnemonic coding of visual space in the monkey's dorsolateral prefrontal cortex. Journal of Neurophysiology, 61, 331-349.

Fuster, J. M., \& Alexander, G. E. (1971). Neuron activity related to short-term memory. Science, 173, 652-654.

Glimcher, P. W., \& Sparks, D. L. (1993). Effects of low-frequency stimulation of the superior colliculus on spontaneous and visually guided saccades. Journal of Neurophysiology, 69, 953-964.

Gold, J. I., \& Shadlen, M. N. (2000). Representation of a perceptual decision in developing oculomotor commands. Nature, 404, 390-394.

Goldman-Rakic, P. S. (1995). Architecture of the prefrontal cortex and central executive. Annals of the New York Academy of Sciences, 769, 71-83.

Goldman-Rakic, P. S. (1996). The prefrontal landscape: Implications of functional architecture for understanding human mentation and the central executive. Philosophical Transactions of the Royal Society of London, Series B, 351, 1445-1453.

Groh, J. M., Born, R. T., \& Newsome, W. T. (1997). How is a sensory map read out? Effects of microstimulation in visual area MT on saccades and smooth pursuit eye movements. Journal of Neuroscience, 17, 4312-4330.

Hanes, D. P., \& Schall, J. D. (1996). Neural control of voluntary movement initiation. Science, 274, 427-430.

Kim, J. N., \& Shadlen, M. N. (1999). Neural correlates of a decision in the dorsolateral prefrontal cortex of the macaque. Nature Neuroscience, 2, 176-185.

Lauwers, K., Saunders, R., Vogels, R., Vandenbussche, E., \& Orban, G. A. (2000). Impairment in motion discrimination tasks is unrelated to amount of damage to superior temporal sulcus motion areas. Journal of Comparative Neurology, $420,539-557$.

Lebedev, M. A., \& Wise, S. P. (2002). More neurons in dorsolateral prefrontal cortex encode spatial attention than encode spatial working memory. Society for Neuroscience Abstracts, 28, 282.8.

Lisberger, S. G., \& Ferrera, V. F. (1997). Vector averaging for smooth-pursuit eye movements initiated by two moving targets in monkeys. Journal of Neuroscience, 17, 7490-7502.

Miller, E. K., \& Cohen, J. D. (2001). An integrative theory of prefrontal cortex function. Annual Review of Neuroscience, 24, 167-202.

Moore, T., \& Armstrong, K. M. (2003). Selective gating of visual signals by microstimulation of frontal cortex. Nature, 421 , 370-373.

Moore, T., \& Fallah, M. (2001). Control of eye movements and spatial attention. Proceedings of the National Academy of Sciences, 98, 1273-1276.

Moore, T., \& Fallah, M. (2004). Microstimulation of the frontal eye field and its effects on covert spatial attention. Journal of Neurophysiology, 91, 152-162.

Newsome, W. T., \& Pare, E. B. (1988). A selective impairment of motion perception following lesions of the middle temporal visual area (MT). Journal of Neuroscience, 8, 2201-2211. 
Nichols, M. J., \& Newsome, W. T. (2002). Middle temporal visual area microstimulation influences veridical judgments of motion direction. Journal of Neuroscience, 22, 9530-9540.

Opris, I., Barborica, A., \& Ferrera, V. P. (2001). On the gap effect for saccades evoked by electrical microstimulation of frontal eye fields in monkeys. Experimental Brain Research, 138, 1-7.

Opris, I., Barborica, A., \& Ferrera, V. P. (2003). Effects of electrical microstimulation of monkey prefrontal cortex in a spatial match-to-sample saccade task. Society of Neuroscience Abstracts, 29, 722.6.

Opris, I., Barborica, A., \& Ferrera, V. P. Effects of electrical microstimulation of monkey prefrontal cortex in a memory-guided saccade task. Submitted for publication.

Pasternak, T., \& Merigan, W. H. (1994). Motion perception following lesions of the superior temporal sulcus in the monkey. Cerebral Cortex, 4, 247-259.

Posner, M. I., \& Cohen, Y. (1984). Components of visual orienting. In H. Bouma and D. G. Bouhuis (Eds.), Attention and performance $X$ (pp. 531-556). London: Erlbaum.

Posner, M. I., Rafal, R. D., Choate, L. S., \& Vaughan, J. (1985). Inhibition of return: Neural basis and function. Cognitive Neuropsychology, 2, 211-228.

Ratcliff, R. (2002). A diffusion model account of response time and accuracy in a brightness discrimination task: Fitting real data and failing to fit fake but plausible data. Psychonomic Bulletin \& Review, 9, 278-291.

Reynolds, J. H., Chelazzi, L., \& Desimone, R. (1999). Competitive mechanisms subserve attention in macaque areas V2 and V4. Journal of Neuroscience, 19, 1736-1753.
Rowe, J. B., Toni, I., Josephs, O., Frankowiak, R. S. J., \& Passingham, R. E. (2000). The prefrontal cortex: Response selection or maintenance within working memory? Science, 288, 1656-1660.

Salzman, C. D., \& Newsome, W. T. (1994). Neural mechanisms for forming a perceptual decision. Science, 264, 231-237.

Schall, J. D., Hanes, D. P., Thompson, K. G., \& King, D. J. (1995). Saccade target selection in frontal eye field of macaque: I. Visual and premovement activation. Journal of Neuroscience, 15, 6905-6918.

Schiller, P. H., \& Chou, I. H. (1998). The effects of frontal eye field and dorsomedial frontal cortex lesions on visually guided eye movements. Nature Neuroscience, 1, 248-253.

Seideman, E., Zohary, E., \& Newsome, W. T. (1998). Temporal gating of neural signals during performance on a visual discrimination task. Nature, 394, 72-75.

Sweeney, J. A., Mintun, M. A., Kwee, S., Wiseman, M. B., Brown, D. L., Rosenberg, D. R., \& Carl, J. R. (1996). Positron emission tomography study of voluntary saccadic eye movements and spatial working memory. Journal of Neurophysiology, 75, 454-468.

Tehovnik, E. J., \& Slocum, W. M. (2003). Microstimulation of macaque V1 disrupts target selection: Effects of stimulation polarity. Experimental Brain Research, 148, 233-237.

Usher, M., \& McClelland, J. L. (2001). The time course of perceptual choice: the leaky, competing accumulator model. Psychological Review, 108, 550-592.

Wang, X.-J. (2002). Probabilistic decision making by slow reverberation in cortical circuits. Neuron, 36, 955-968. 